\title{
Débat avec les responsables scientifiques de l'axe 3
}

\section{Valérie Carayol et Paul Stryckman}

\section{OpenEdition}

Journals

Édition électronique

URL : http://journals.openedition.org/communicationorganisation/2005

DOI : 10.4000/communicationorganisation.2005

ISSN : $1775-3546$

\section{Éditeur}

Presses universitaires de Bordeaux

\section{Édition imprimée}

Date de publication : 1 novembre 1997

ISSN : 1168-5549

\section{Référence électronique}

Valérie Carayol et Paul Stryckman, «Débat avec les responsables scientifiques de l'axe 3 », Communication et organisation [En ligne], 12 | 1997, mis en ligne le 26 mars 2012, consulté le 19 avril 2019. URL : http://journals.openedition.org/communicationorganisation/2005 ; DOI : 10.4000/ communicationorganisation.2005

Ce document a été généré automatiquement le 19 avril 2019

(c) Presses universitaires de Bordeaux 


\title{
Débat avec les responsables scientifiques de l'axe 3
}

\author{
Valérie Carayol et Paul Stryckman
}

1 Valérie Carayol: Vous avez dit que "dans la mêlée du direct, nous participons plutôt que nous symbolisons» et que "l'induction se vit au présent». Hier, avec Wolfgang Settekorn qui nous a parlé de métaphorisations mutuelles avec des exemples visuels et avec Philippe Breton qui nous a parlé d'amalgame, on avait déjà esquissé un rapprochement entre l'induction et les dynamiques spatiales, pas obligatoirement une dynamique temporelle. Est-ce que vous pourriez préciser cette idée du direct, de la simultanéité dans le temps?

2 Freud dit que l'inconscient est time-less, il ignore la temporalité. Il me semble que quand on vit l'induction, les frontières de l'espace et du temps, le cadre spatio-temporel, est profondément remanié pour ne pas dire aboli. Bien sûr on est jamais hors-temps, ni horsespace. Mais nous savons tous que parfois le temps ne passe pas ou passe trop vite. L'espace peut être plastique et soumis aux désirs et fantasmes ou à l'intersubjectivité des partenaires d'une relation forte. Donc, je pense que ces cadres spatio-temporels sont éminemment déformables et que le symbolisme s'efforce de recadrer cela de façon stable ; après tout quand on tient le chronomètre comme le président de séance, il y a cet ordre symbolique immuable, néanmoins il y a des plages, des moments de participation et d'induction chaudes où le cadre symbolique est effondré pour chacun, mais cela revient à se les rappeler. Le présent de l'induction me semble un de ces effondrement symboliques intéressant à cadrer. Il faut parler de cela, il faut le remarquer, bien sûr nous le vivons, nous n'allons pas le dire, chacun le sait et le vit. On a du mal après-coup à le remettre dans la chronologie, dans la marqueterie de nos représentations ordinaires, parce que c'est le trou dans le vécu. L'intensité, le présent vécu fait trou dans l'histoire, dans la trame et en tout cas dans le symbolique.

3 Valérie Carayol: Quelque chose m'a séduit aussi dans ce que vous avez dit. Vous avez parlé à propos de l'induction, de communication primaire, que j'ai interprétée comme étant la communication du moindre effort. Est-ce qu'on pourrait parler avec l'induction d'une communication qui fonctionnerait au principe d'économie. On parle de court-circuit, de 
constellations d'effets, de choses immédiates, de processus primaires. Je voulais vous interroger sur ce principe d'économie ou de communication du moindre effort.

Bien sûr, c'est le principe de plaisir comme dit Freud. Mais, il explique le plaisir par l'économie, dans le grand texte sur les mots d'esprit. Quel est le plaisir que nous prenons dans les mots d'esprit? C'est un plaisir d'épargne psychique, on appelle cela l'économie. L'explication économique est constante dans le texte des mots d'esprit, selon Freud. Or, c'est un court-circuit, par rapport à la chaîne argumentative. Ce qui fait que quelqu'un qui argumente face à quelqu'un qui fait des mots d'esprit aura toujours le dessous. Une communauté de rieurs est inattaquable comme telle. On ne peut nullement s'approcher de rieurs avec une argumentation secondaire, on est battu d'avance; de même avec les communautés affectives. C'est un pléonasme, on pourrait dire communauté, parce que le plus sûr ciment d'une communauté c'est peut-être le court-circuit primaire qui fait qu'on s'absente d'un effort secondaire, d'une élaboration plus longue, plus coûteuse et par définition plus pénible. Je suis très frappé par cette alternance du primaire et du secondaire, mais je ne voudrais pas qu'on secondarise le primaire et qu'on aborde cela avec la catégorie du haut de la pile. Il y a en nous en permanence la présence des processus primaires et cette abîme du processus primaire. Pour le meilleur et pour le pire. Le processus primaire, c'est la jubilation, l'amour, la fusion. C'est l'absence d'espace et de temps. Bien-sûr c'est en même temps la psychose, le sommeil, à la fois des choses positives et négatives. En fait, nous alternons l'un et l'autre, nous corrigeons l'un à l'autre.

5 Paul Stryckman: Je vais interpeller Monsieur Bougnoux et être prudent en l'invitant à répondre à sa propre invitation. C'est-à-dire travailler sur l'intelligence du lien entretenu lors $d u$ rendez-vous dans la crypte; on a compris qu'il y a deux temps qui se fondent dans un travail assimilateur entre présent, et passé, projet et récit, si on prend la terminologie de Boris Cyrulnik. Tout d'abord, il y a le non-roman. Ce que vous appelez la stabilité, le caisson, le phénomène traumatisant qui ne peut pas êre travaillé ou qui serait plus fort que moi, et qui se laisse reconnaitre de façon infra-verbale. Alors sur cette base, je poserai deux questions. On pourrait passer de la métaphore de la crypte à celle du trou noir, en nous demandant tout simplement ce qu'on fait du concept flou et donc nécessairement mobilisateur d'un nationalisme latent. Est-ce que cette notion là peut habiter la crypte? Comment faire une analyse sémantique de cette notion de secret? Dans quelle mesure le secret ne peut pas être public? Je pense aux événements vécus récemment au Québec, où tout le monde n'était pas dans la même crypte.

6 Dans la logique, de cette question là, je poserai un point occulté dans l'ensemble de notre démarche: l'espace langagier. Dans quelle mesure les pratiques langagières en tant que déclinaisons dures de plusieurs langues ne seraient pas des problèmes importants en tant que prétexte ou pré-énoncé qui assurent une autorité à la parole et en même temps lui donne les pleins pouvoirs; en ce sens qu'il y aurait, pour reprendre l'image de Sapir, des partitions secrètes ou ignorées qui modulent des accents. Dans le cas d'une bilingualité, cas typique de société comme Montréal, nous avons un problème très dense. Peut-on réduire le langage, ce problème de langue de départ comme un simple problème de symbole ou bien il y a une récupération, en disant qu'il y a une symbolisation inhérente de la langue elle-même comme facteur d'autorité et de reconnaissance de l'identité.

7 On s'interroge ici sur l'en deçà du symbolique dans l'en deçà du linguistique et sur ce qui fait lien autrement que culturellement de façon un peu superficielle entre nous, de façon un peu trop cohérente entre nous. Il faut absolument quitter cette grande moyenne des phénomènes en communication et il faut plonger dans l'envers, dans l'enfer, dans l'infra. 
L'enfer c'est la crypte, c'est l'indiciel, le charnel. Ce sont les tombes, les morts, les crimes, les secrets partagés ou non par les membres d'une communauté.

J'ai toujours été frappé par la faible charge mythologique des théories de Rousseau ou des Lumières concernant le contrat social. Il est certain que notre siècle a infligé le plus cinglant démenti à ces sociétés conçues sur le modèle du contrat. On a vu le retour en force des communautés chaudes, et encore aujourd'hui l'islamisme, les éthnies sur le continent nord-américain où le communautarisme est très fort. Des fractures linguistiques, des fractures culturelles apparaissent y compris sur le campus, et au sein même des enseignements qui devraient être le lieu de la raison, des échanges, et de la communication culturelle et qui, au contraire, sont des lieux d'oppositions et de fractures culturelles. Il y a un phénomène qui est entre nous, sous nos yeux, qu'on ne pourra pas traiter avec des considérations de communications ordinaires. Je crois qu'il faut plonger dans ce que Freud pointe comme une processus primaire, même si la psychanalyse parfois recule devant ses propres découvertes (elle recadre ou replaque le primaire, ou le tribal, ou l'hypnotique ou la masse par des catégories secondaires).

9 À mon avis, nos sciences devraient aller dans ce sens. J'étudie cela depuis peu de temps et je suis pas trop sûr de ce que je vais dire maintenant. Je sens qu'il y a là une chose très forte. On communique bien par les tombes par exemple et par les secrets de famille qui créent des pactes entre les gens. Le pacte est très en-décela du contrat. Dans le pacte, il y a quelque chose de charnel; c'est plus que familial. C'est cette masse primaire que peut constituer une famille. Il faut plonger dans la relation mère-enfant, dans la relation de langue maternelle, de nourrisson, parce que le linguistique touche aussi par la langue maternelle à des rapports de succion, d'articulation chamelle de la langue au grand corps qui est celui de la mère, de la marrie, de la patrie, etc.. Il faut redonner leur poids de chair à ces notions trop symboliques, trop linguistiques, trop culturelles. Et là, le structuralisme a été un peu léger peut-être. Quand on reprend les dossiers des grands totalitarismes, aujourd'hui le dossier du politiquement correct, les fractures et retours d'éthnies, on ne peut pas rester à la surface de la bande moyenne de la communication. Je ne peux pas en dire plus sur le jeu de communautés chaudes organiques, mais il est certain que le siècle a offert trop d'exemples de cela et encore aujourd'hui c'est devant nous. Il faut affronter et cadrer cela. D'autant plus que les sorciers jouent admirablement bien avec cela.

10 Je suis frappé par le danger et les ressources de plaisir qu'il y a dans cette communication primaire. C'est un peu pour cela que j'ai écrit la communication contre l'information, c'était pour protester contre les visions trop moyennes des phénomènes que nous étudions, trop théoriques. Il n'y a pas que l'information dans la communication.

\section{AUTEURS}

\section{VALÉRIE CARAYOL}

Université Michel de Montaigne-Bordeaux 3, ISIC-IUP 


\section{PAUL STRYCKMAN}

Université Laval, (Québec) Département Information et communication 